\title{
Nonlinear response via intrinsic rotation in metamaterials
}

\author{
Mingkai Liu, ${ }^{1,}$ Yue Sun, ${ }^{1}$ David A. Powell, ${ }^{1}$ Ilya V. Shadrivov, ${ }^{1}$ Mikhail Lapine, ${ }^{2}$ Ross C. McPhedran, ${ }^{2}$ and Yuri S. Kivshar ${ }^{1}$ \\ ${ }^{1}$ Nonlinear Physics Centre and Centre for Ultrahigh-bandwidth Devices for Optical Systems (CUDOS), Research School of Physics and \\ Engineering, Australian National University, Canberra ACT 0200, Australia \\ ${ }^{2}$ Centre for Ultrahigh-bandwidth Devices for Optical Systems (CUDOS), School of Physics, University of Sydney, Sydney NSW 2006, Australia
}

(Received 7 November 2012; published 20 June 2013)

\begin{abstract}
We propose and experimentally verify a way to achieve strong nonlinear coupling between the electromagnetic and elastic properties in metamaterials. This coupling is provided through a novel degree of freedom in metamaterial design: the internal rotation within structural elements. Our meta-atoms have high sensitivity to electromagnetic wave power, and the elastic and electromagnetic properties can be independently designed to optimize the response. We demonstrate a rich range of nonlinear phenomena including self-tuning and bistability, and provide a comprehensive experimental demonstration of the predicted effects.
\end{abstract}

DOI: 10.1103/PhysRevB.87.235126 PACS number(s): 81.05.Xj, 05.45.-a, 42.65.Pc, 78.67.Pt

\section{INTRODUCTION}

Metamaterials research has grown rapidly over the past decade, exhibiting a wide variety of new wave phenomena. ${ }^{1,2}$ Being initially conceived in the domain of electromagnetics, ${ }^{3-5}$ the metamaterial concept also proved to be fruitful in other areas of physics. ${ }^{6-8}$ Until recently, however, direct interplay between different types of physical effects within the same metamaterial was not considered, although mechanical control over electromagnetic metamaterial properties was employed in structural tuning. ${ }^{9,10}$

It turns out that introducing a mechanical degree of freedom into electromagnetic metamaterials leads to an interesting range of nonlinear effects, giving rise to a new class of magnetoelastic metamaterials ${ }^{11}$ and to wide-band operation. ${ }^{12}$ The range of possible effects achievable in this way promises to be richer than in the prominent area of optomechanics, ${ }^{13}$ because the greater flexibility in metamaterial design overcomes the limits of available material functionalities and offers wider possibilities for optimization. At the same time, the implementation of magnetoelastic metamaterials ${ }^{11}$ remains challenging and in some cases, such as the conformational nonlinearity in resonant spirals, ${ }^{14}$ remains inaccessible for optics. The reason for this is that the magnetic forces, employed in the initial designs, are relatively weak, so such materials require either high power or extremely small elastic restoring forces, which poses a considerable manufacturing challenge.

We recall, however, that earlier research on structurally tunable metamaterials ${ }^{9}$ indicated that near-field interaction may significantly improve the tunability range, leading to various effects associated with near-field coupling. ${ }^{15}$ In particular, changing the mutual orientation between the neighboring elements has a profound effect on the resonator coupling and the structure of their modes. ${ }^{16,17}$

We therefore expect that the most efficient approach to implement dynamic coupling between electromagnetic and mechanical effects should rely on the near-field interaction, which can have a powerful influence even for subtle changes in the mutual orientation. Instead of a considerable displacement of an entire array, ${ }^{9}$ it is sufficient to move the crucial parts of the resonant particles with respect to each other-for example, the gaps of the two coupled split-ring resonators-which involves a more gentle geometric alteration.

\section{DESIGN AND GENERAL CONCEPT}

Let us now introduce our concept: nonlinear metamaterials with intrinsic rotation. As a building element of the structure (see Fig. 1), we consider two coaxial split ring resonators (SRRs) with elastic feedback between them. The rings are allowed to rotate about the common axis, while the elastic feedback is provided by connecting a thin elastic wire. Indeed, the use of elastic wires has a prominent history in physics, being utilized in the milestone achievement of the experimental demonstration of light pressure by P. N. Lebedev. ${ }^{18}$ Here, we employ the electromagnetic (EM) torque to construct a "lightdriven" meta-atom, which enables us to modulate the resonant frequencies by twisting the rotatable element directly with EM waves. The component of the EM forces, which twists the rings with respect to each other, is normally not the strongest among the forces involved, but the prominent advantage of using EM torque instead of collinear EM force to drive a meta-atom is that the effective lever arm of the azimuthal EM force can be much larger than that of the azimuthal restoring force from a thin wire; this can effectively magnify the deformation in azimuthal direction by orders of magnitude.

Technically, there are a number of ways to implement this general scheme; in our design, one of the rings is fixed to a substrate and the other one is suspended on the long wire. The three symmetrically positioned wires, which attach the suspended ring to the string provide stability against tilt. In this design, therefore, the only favorable movement is the rotation of the suspended ring with respect to the fixed ring over the common axis, and all other mechanical degrees of freedom can be neglected.

Suppose the initial position is such that the ring slits have a certain angle between them with respect to the common axis (see Fig. 1). An EM wave then induces a certain distribution of charges and currents in the two resonators, and the resonance of the system is determined by their mutual orientation. ${ }^{16}$ These charges and currents also result in EM torque between the two rings, ${ }^{19}$ which drives the suspended ring to rotate until the EM torque is compensated by the elasticity of the twisted wire. Meanwhile, the entire pattern of charges and currents gets modified and the torque also changes, so the final stable equilibrium is only achieved via a complex nonlinear feedback. The twisted wire provides a restoring torque to balance the 

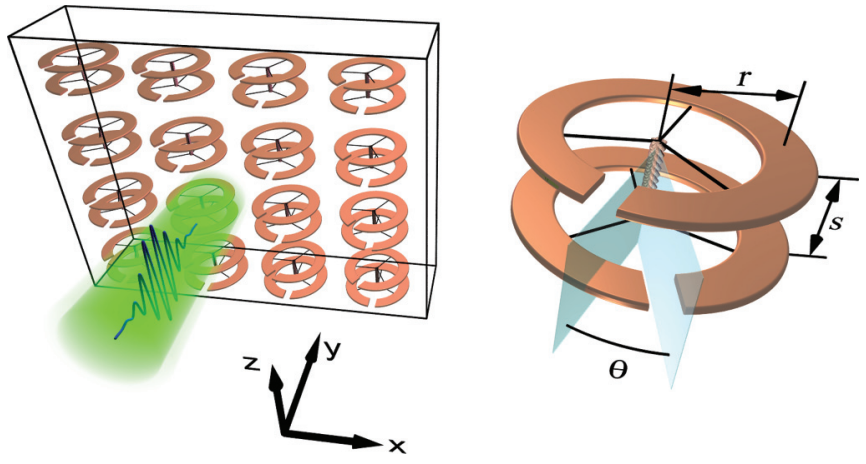

FIG. 1. (Color online) Conceptual layout of a new metamaterial and its rotational "meta-atom." Incident wave propagates along $y$ direction, having a linear polarization with the electric field along $x$ and magnetic field along $z$. The induced electromagnetic torque between the resonators changes the mutual twist angle $\theta$ between the rings, connected by an elastic wire.

EM torque, so that we can control the twist angle (and thus the resonance) by changing the external field.

An additional feature of the proposed design is that the nonlinear dynamics of the rotatable particle not only depend on the parameters of the wire, but also on the EM mode initially excited in the resonators, which is determined by the starting angle between the gaps. The latter can be made arbitrary, and an implementation has the possibility to deliberately adjust it, introducing tunability to the system.

The above design, therefore, offers a tunable resonant nonlinear system with elastic feedback and, as we show below, yields a rich pattern of nonlinear response including selftuning and nonlinear bistability, which are much stronger than those provided by using nonlinear semiconductor components. Below, we present a detailed theoretical analysis of rotational meta-atoms. We then proceed to the experimental results obtained with a fabricated prototype of the rotational "metaatom" placed in a rectangular waveguide, and confirm all important features predicted by theory. Finally, we perform full-wave numerical simulations of the array of "meta-atoms" (i.e., metamaterial) and demonstrate that all the nonlinear effects observed for the single meta-atom in the waveguide are qualitatively the same in the array.

\section{THEORETICAL ANALYSIS}

\section{A. Semianalytical model}

To start with, we use a semianalytical model to study the dynamics of an isolated meta-atom in free space. It will subsequently be demonstrated that this model explains all qualitative features of an array. As shown in Fig. 1, the two coaxial identical SRRs are offset by a distance $s$ in the $z$ direction, and the twist angle between them is $\theta$. The incident wave propagates along the $y$ direction, with its magnetic field in the $z$ direction and electric field in the $x$ direction. The orientation of the gaps with respect to the incident electric field polarization is described by the angle $\Phi$ between the azimuth of the gap of the bottom ring as seen from the ring axis and $y$ axis.

To study the nonlinear behavior of the rotatable meta-atom, we utilize an efficient analytical model based on the single mode approximation and the near-field interaction. ${ }^{16}$ This model can provide a reasonable prediction of the EM response as well as the optomechanical properties of the structures. ${ }^{19}$ As our previous studies showed, ${ }^{20}$ the current and charge of the SRR can be separated into frequency-dependent mode amplitudes and spatially dependent distributions: $\mathbf{J}(\mathbf{r}, \omega)=$ $-j \omega Q(\omega) \mathbf{j}(\mathbf{r})$ and $\rho(\mathbf{r}, \omega)=Q(\omega) q(\mathbf{r})$. The mode amplitudes $Q_{1,2}$ can be obtained after solving the coupled equations:

$$
\begin{aligned}
& Q_{1}=\left(\mathcal{E}_{2} F_{m}-\mathcal{E}_{1} F_{s}\right) /\left(F_{s}^{2}-F_{m}^{2}\right), \\
& Q_{2}=\left(\mathcal{E}_{1} F_{m}-\mathcal{E}_{2} F_{s}\right) /\left(F_{s}^{2}-F_{m}^{2}\right),
\end{aligned}
$$

where $\mathcal{E}_{1}$ and $\mathcal{E}_{2}$ correspond to the effective voltage applied to the lower (index 1) and top (index 2) SRRs by the external fields:

$$
\mathcal{E}_{1}=-\mathbf{E}_{\mathrm{ext}} \cdot \mathbf{l}_{e} \cdot e^{j k_{0} a_{E} \cos \Phi}+j \omega \mathbf{B}_{\mathrm{ext}} \cdot \mathbf{u}_{e} \cdot e^{j k_{0} a_{M} \cos \Phi}
$$

and

$\mathcal{E}_{2}=-\mathbf{E}_{\mathrm{ext}} \cdot \mathbf{l}_{e} \cdot e^{j k_{0} a_{E} \cos (\Phi+\theta)}+j \omega \mathbf{B}_{\mathrm{ext}} \cdot \mathbf{u}_{e} \cdot e^{j k_{0} a_{M} \cos (\Phi+\theta)}$.

The normalized electric dipole moment is $\mathbf{l}_{e}(\theta, \Phi)=$ $\int_{V} q(\mathbf{r}) \mathbf{r} \mathrm{d} V$, and $\mathbf{u}_{e}(\theta, \Phi)=\frac{1}{2} \int_{V} \mathbf{r} \times \mathbf{j}(\mathbf{r}) \mathrm{d} V$ is the normalized magnetic dipole moment.

We define the effective central positions of the electric and magnetic dipoles

$$
\begin{gathered}
a_{E}=\frac{\int_{V}\left[q\left(\mathbf{r}_{1}\right) \mathbf{r}_{1} \cdot \hat{\mathbf{x}}\right]\left(\mathbf{r}_{1} \cdot \hat{\mathbf{y}}\right) \mathrm{d} V_{1}}{\left|\int_{V} q\left(\mathbf{r}_{1}\right) \mathbf{r}_{1} \mathrm{~d} V_{1}\right|}, \\
a_{M}=\frac{\int_{V}\left[\mathbf{r}_{1} \times \mathbf{j}\left(\mathbf{r}_{1}\right) \cdot \hat{\mathbf{z}}\right]\left(\mathbf{r}_{1} \cdot \hat{\mathbf{y}}\right) \mathrm{d} V_{1}}{\left|\int_{V} \mathbf{r}_{1} \times \mathbf{j}\left(\mathbf{r}_{1}\right) \mathrm{d} V_{1}\right|}
\end{gathered}
$$

similar to the definition of center of mass, and they are calculated based on the charge and current distributions of the lower SRR when $\Phi=0$. The phase terms of the effective voltages describe the phase retardation experienced by the SRRs in the direction of wave propagation. $F_{s}=1 / C_{s}-\omega^{2} L_{s}$ and $F_{m}=1 / C_{m}-\omega^{2} L_{m}$ are the self- and mutual impedance terms, where the effective capacitances $C$ and inductances $L$ can be calculated from the modal current $\mathbf{j}(\mathbf{r})$ and charge $q(\mathbf{r})$ distributions (see Ref. 20).

Once the frequency-dependent mode amplitudes are known, we can calculate the EM torque experienced by the SRRs. Here, we are particularly interested in the torque on the top rotating ring: $\mathbf{M}_{\mathrm{EM}}=\int_{V_{2}} \rho\left(\mathbf{r}_{2}\right) \mathbf{r}_{2} \times \mathbf{E}+\mathbf{r}_{2} \times\left[\mathbf{J}\left(\mathbf{r}_{2}\right) \times\right.$ $\mathbf{B}] \mathrm{d} V_{2}$, where the integration is performed over the volume $V_{2}$ of the top SRR. We decompose the total torque into two parts: external torque $\mathbf{M}_{\mathrm{ext}}$ contributed by the external incident fields, ${ }^{19}$ and internal torque $\mathbf{M}_{\text {int }}$ due to the near-field interaction between the two SRRs. We assume that the lower SRR is fixed while the top SRR is only allowed to rotate about the $z$ axis. The explicit expressions for the internal and external torque are as follows:

$$
\begin{aligned}
& \mathbf{M}_{\text {ext }, 2}=\frac{1}{2} \operatorname{Re}\left[\int_{V_{2}} \rho^{*}\left(\mathbf{r}_{2}\right) \mathbf{r}_{2} \times \mathbf{E}_{\text {ext }} \mathrm{d} V_{2}\right] \\
& =-\frac{1}{2} \operatorname{Re}\left[Q_{2}^{*}(\omega, \Phi) e^{j k_{0} a_{E} \cos (\Phi+\theta)}\right] \mathbf{E}_{\text {ext }} \cdot \mathbf{l}_{\mathrm{e}} \sin (\Phi+\theta) \cdot \hat{\mathbf{z}},
\end{aligned}
$$


while the internal torque can be expressed as

$$
\mathbf{M}_{\text {int }, 2}=\frac{1}{2} \operatorname{Re}\left[\int_{V_{2}} \rho^{*}\left(\mathbf{r}_{2}\right) \mathbf{r}_{2} \times \mathbf{E}_{\text {int }}\left(\mathbf{r}_{2}\right) \mathrm{d} V_{2}\right]
$$

with

$$
\begin{aligned}
\mathbf{E}_{\text {int }}\left(\mathbf{r}_{2}\right) & =-\nabla \phi\left(\mathbf{r}_{2}\right)-\frac{\partial}{\partial t} \mathbf{A}\left(\mathbf{r}_{2}\right) \\
& =-\int_{V_{1}} \nabla \frac{\rho\left(\mathbf{r}_{1}\right) e^{j k\left|\mathbf{r}_{2}-\mathbf{r}_{1}\right|}}{4 \pi \epsilon_{0}\left|\mathbf{r}_{2}-\mathbf{r}_{1}\right|}+\frac{\partial}{\partial t} \frac{\mathbf{J}\left(\mathbf{r}_{1}\right) e^{j k\left|\mathbf{r}_{2}-\mathbf{r}_{1}\right|}}{4 \pi c^{2} \epsilon_{0}\left|\mathbf{r}_{2}-\mathbf{r}_{1}\right|} \mathrm{d} V_{1} .
\end{aligned}
$$

Finally, we arrive at

$$
\begin{aligned}
\mathbf{M}_{\mathrm{int}, 2}= & \frac{1}{2} \operatorname{Re}\left\{\frac{Q_{1}(\omega) Q_{2}^{*}(\omega)}{4 \pi \epsilon_{0}} \iint \frac{q^{*}\left(\mathbf{r}_{2}\right) e^{j k\left|\mathbf{r}_{1}-\mathbf{r}_{2}\right|}}{\left|\mathbf{r}_{1}-\mathbf{r}_{2}\right|}\right. \\
& \times\left[\frac{1-j k\left|\mathbf{r}_{1}-\mathbf{r}_{2}\right|}{\left|\mathbf{r}_{1}-\mathbf{r}_{2}\right|^{2}} q\left(\mathbf{r}_{1}\right) \mathbf{r}_{1} \times \mathbf{r}_{2}+k^{2} \mathbf{r}_{2} \times \mathbf{j}\left(\mathbf{r}_{1}\right)\right] \\
& \left.\times \mathrm{d} V_{1} \mathrm{~d} V_{2}\right\}
\end{aligned}
$$

For a cylindrical wire that can be used to suspend the rotatable SRR, the restoring torque can be estimated as $M_{R}=-\pi a^{4} G\left(\theta-\theta_{0}\right) /(2 d)$, where $a$ and $d$ are the radius and the length of the wire, respectively. $G$ is the shear modulus and $\theta_{0}$ is the initial twist angle of the structure. A series of equilibrium twist angles can be found by solving the nonlinear equation

$$
M_{\mathrm{EM}}\left(\theta, P_{\mathrm{I}}\right)+M_{R}\left(\theta, \theta_{0}\right)=0 .
$$

\section{B. Numerical example}

We model a pair of twisted SRRs, with radius $r=6 \mathrm{~mm}$ and vertical spacing $s=2 \mathrm{~mm}$. The size of the slit in each ring can be described by the angle at which the gap is visible from the ring center, $\alpha_{0}=10^{\circ}$. The elastic coupling is provided with a wire of radius $a=50 \mu \mathrm{m}$ and of length $d=100 \mathrm{~mm}$, made from rubber with a shear modulus of $G=0.6 \mathrm{MPa}$.

Figures 2(a) and 2(b) depict the mode amplitude $Q_{2}$ and the total EM torque $\mathbf{M}_{\mathrm{EM}}=\mathbf{M}_{\mathrm{ext}}+\mathbf{M}_{\text {int }}$ experienced by the top SRR as functions of frequency and twist angle $\theta$. In our dimer meta-atom, changing $\Phi$ generally leads to some difference in the coupling efficiency between the input plane wave and the eigenmodes, but does not cause any substantial qualitative difference such as the direction of EM torque, since it is a property governed by the symmetry of the mode profiles. Without loss of generality, we have assumed $\Phi=0$ for the calculations. Since radiation losses are taken into account in the model, ${ }^{20}$ we are able to accurately describe the evolution of the mode amplitudes, phases and line shapes of the resonances. As expected, this chiral meta-atom supports two hybrid resonances, which can be characterized as symmetric (lower frequency branch) and antisymmetric (higher frequency branch) modes, according to the symmetry of the $H_{z}$ component. $^{16}$

The directions of the EM torque at these two resonances are also opposite. For the symmetric mode, $\theta=0^{\circ}$ corresponds to the configuration of highest potential energy (unstable point), and thus the two repel each other once $\theta>0^{\circ}$, until they come
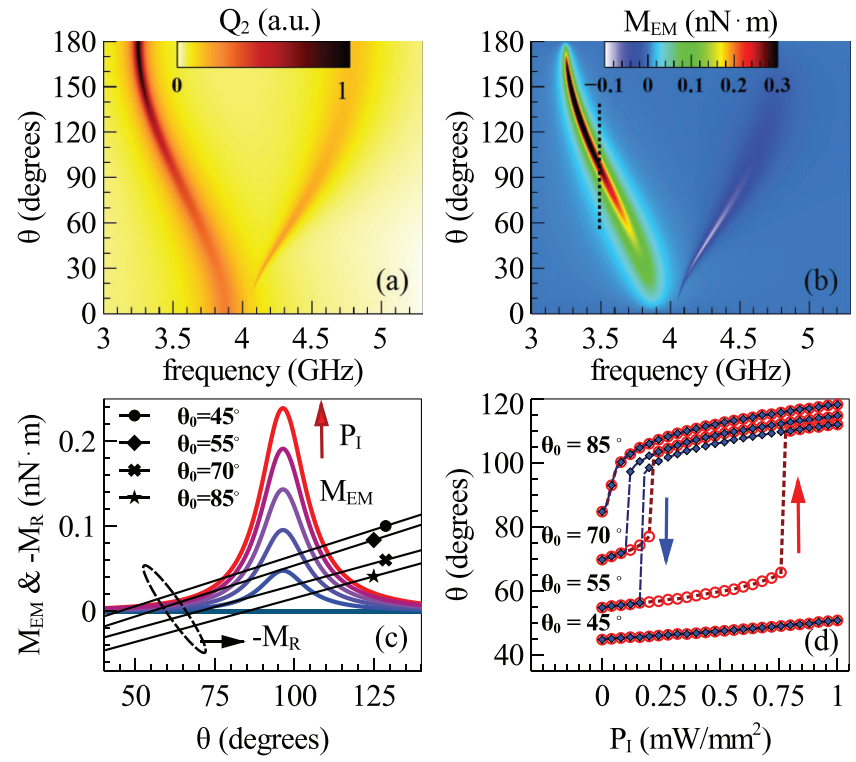

FIG. 2. (Color online) The principle of nonlinear response in rotatable meta-atoms. (a) The mode amplitude $Q_{2}$ and (b) the EM torque $\mathbf{M}_{\mathrm{EM}}$ of the top rotatable ring. (c) The EM torque at $3.5 \mathrm{GHz}$ for different pump powers from 0 to $1 \mathrm{~mW} / \mathrm{mm}^{2}$ in $0.2 \mathrm{~mW} / \mathrm{mm}^{2}$ steps, and the restoring torque for different initial twist angles $\theta_{0}$; (d) the corresponding paths of power-dependent twist angles under different $\theta_{0}$.

to the stable state at $\theta=180^{\circ}$, while the reverse is true for the antisymmetric mode. ${ }^{21}$ The evaluated external torque is about one order of magnitude smaller than the internal torque, and the total torque is of the order of $10^{-10} \mathrm{Nm}$ when the structure is pumped with a power density $P_{\mathrm{I}}=1 \mathrm{~mW} / \mathrm{mm}^{2}$. This is confirmed by the full-wave simulation (CST Microwave Studio) followed by calculation based on the Maxwell stress tensor, which yields the EM torque through a surface integral of the field components around the object. ${ }^{22}$

The overall mechanism of achieving a nonlinear effect is presented in Figs. 2(c) and 2(d). As an example, we choose a pump frequency $(3.5 \mathrm{GHz})$ at the symmetric mode [regime denoted by the black dashed line in Fig. 2(b)]. It can be seen that $\mathbf{M}_{\mathrm{EM}}$ is a Lorentz-like function of the twist angle, while the restoring torques $\mathbf{M}_{R}$ under different initial twist angles $\theta_{0}$ are approximated by linear functions (Hooke's law). The intersections of these two functions, $\mathbf{M}_{\mathrm{EM}}\left(\theta_{e}, P_{\mathrm{I}}\right)+\mathbf{M}_{R}\left(\theta_{e}\right)=$ 0 , correspond to the equilibrium angles $\theta_{e}$. However, only the angles with $\frac{\partial}{\partial \theta}\left[\mathbf{M}_{\mathrm{EM}}\left(\theta_{e}\right)+\mathbf{M}_{R}\left(\theta_{e}\right)\right]<0$ are stable. As the pump power $P_{\mathrm{I}}$ increases from zero to maximum and then reduces, the stable angles also change accordingly. With this method, we can numerically find a sequence of stable angles under different pump power $P_{\mathrm{I}}$.

Since the EM torque is a nonlinear function of twist angle, it naturally leads to nonlinear solutions. As shown in Fig. 2(d), the power-dependent twist angles under different initial angles demonstrate the evolution from smooth nonlinear to bistable response as $\theta_{0}$ departs from the angle of maximum EM torque. In principle, as $\theta_{0}$ moves further away from the resonance, more noticeable rotation and hysteresis effects are expected, but higher pump power is required (see the case for $\theta_{0}=45^{\circ}$ ). Such evolution of the power-dependent nonlinear response can 
also be observed by fixing the initial twist angle but changing the pump frequency, as will be demonstrated in the experiment below.

\section{EXPERIMENTAL VERIFICATION}

\section{A. Experimental setup and measurement}

To confirm the feasibility of the proposed nonlinear rotatable meta-atoms, we carry out a pump-probe microwave experiment. To experimentally realize a strong nonlinear or even bistable effect, the restoring torque from the wire has to be sufficiently small so that the structure can be twisted by a large enough angle within the maximum available power. We found that rubber is a good candidate, since the shear modulus of rubber is of the order of $0.2-2.4 \mathrm{MPa},{ }^{23}$ which is at least three orders of magnitude smaller than it is for other polymers.

A schematic of the experimental setup is shown in Fig. 3. We have used two separated copper SRRs (inner radius $r=3.2$ $\mathrm{mm}$, track width $1 \mathrm{~mm}$, copper thickness $0.035 \mu \mathrm{m}$, and slit width $g=0.2 \mathrm{~mm}$ ) printed on Rogers R4003 substrates $\left(\epsilon_{\mathrm{r}}=3.5\right.$, loss tangent 0.0027 , substrate thickness $\left.0.5 \mathrm{~mm}\right)$. The lower SRR is fixed and positioned at the center of a WR229 rectangular waveguide with $\Phi=0^{\circ}$, and the top SRR is suspended with a thin rubber wire (radius $a=50 \mu \mathrm{m}$, length $d=20 \mathrm{~mm}$ ), so that it can rotate about the common axis. The two SRRs are aligned coaxially, with a face to face distance of $0.75 \mathrm{~mm}$, separated by air. The horizontal positions of the SRRs are carefully adjusted and the initial twist angle $\theta_{0}$ is set at around $70^{\circ}$. The mass of the suspended sample is $101 \mathrm{mg}$, which leads to a $6.1 \%$ elongation of the wire. The Young's modulus is thus estimated as $2.06 \mathrm{MPa}$, and the shear modulus follows as $G \approx 0.69 \mathrm{MPa}$.

Since the mechanism of nonlinear response is the dynamic coupling between electromagnetic and elastic properties, the experimental setup exhibits the same physics as the conceptual schematic although it is slightly different. In the experiment, it was not necessary to fix the bottom end of the wire to the substrate, because the maximum forces emerging in the lateral directions $(\sim 0.1 \mu \mathrm{N}$ according to our calculations $)$ can only produce a negligible swing angle $\left(0.056^{\circ}\right)$ since they are much smaller than the weight of the sample $(\sim 980 \mu \mathrm{N})$. The calculated torque is a Lorentz-like function and the maximum

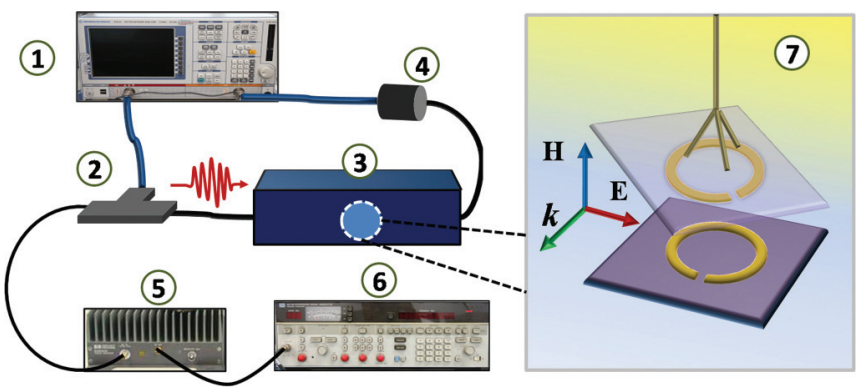

FIG. 3. (Color online) Schematic of the experimental setup. The pump and probe signals are combined by a $3-\mathrm{dB}$ combiner. The sample is positioned in the center of the waveguide. (1) Vector network analyzer, (2) 3-dB combiner, (3) rectangular waveguide, (4) 20-dB attenuator, (5) power amplifier, (6) signal generator, and (7) sample. is around $0.8 \mathrm{nNm}$. The maximum attractive force between the two SRRs is around $2 \mu \mathrm{N}$, which leads to a negligible elongation of the wire $(0.012 \%)$; while the force in the lateral direction is around $0.1 \mu \mathrm{N}$, about 1000 times smaller than the gravitational force exerted on the sample, and thus it only gives rise to a tiny swing angle $\left(0.056^{\circ}\right)$.

The transmission spectrum measurements are performed by a vector network analyzer (Rohde and Schwarz ZVB-20). The CW pump signal is generated by a signal generator (HP 8673B) and is further amplified by a power amplifier (HP 83020A) before being sent into the waveguide. Three pump frequencies $(3.18,3.21$, and $3.23 \mathrm{GHz})$ are chosen in order to capture the evolution of the nonlinear response at different distances from the initial resonance. The pump power is increased in 1-dB steps; for each step, the sample reaches steady state after $30 \mathrm{~s}$. The mechanical rotation is quite significant and can be visually observed in the experiment, thus ruling out other possible nonlinear mechanisms such as heating.

The experimentally observed transmission spectra are shown in Fig. 4, and the corresponding resonant frequencies are depicted in Figs. 5(a), 5(c), and 5(e), where the predicted evolution from bistability to smooth nonlinearity is clearly shown. The initial resonance (symmetric mode) without pump is located around $3.256 \mathrm{GHz}$, and it redshifts as the pump power increases, which indicates that the twist angle is increased. When the pump frequency is at the red tail of the resonance, a large spectral "jump" (about three times of the resonance linewidth) can be observed when the pump power passes a certain threshold value [see Fig. 5(a)]. The thresholds are different for increasing and decreasing pump powers. As
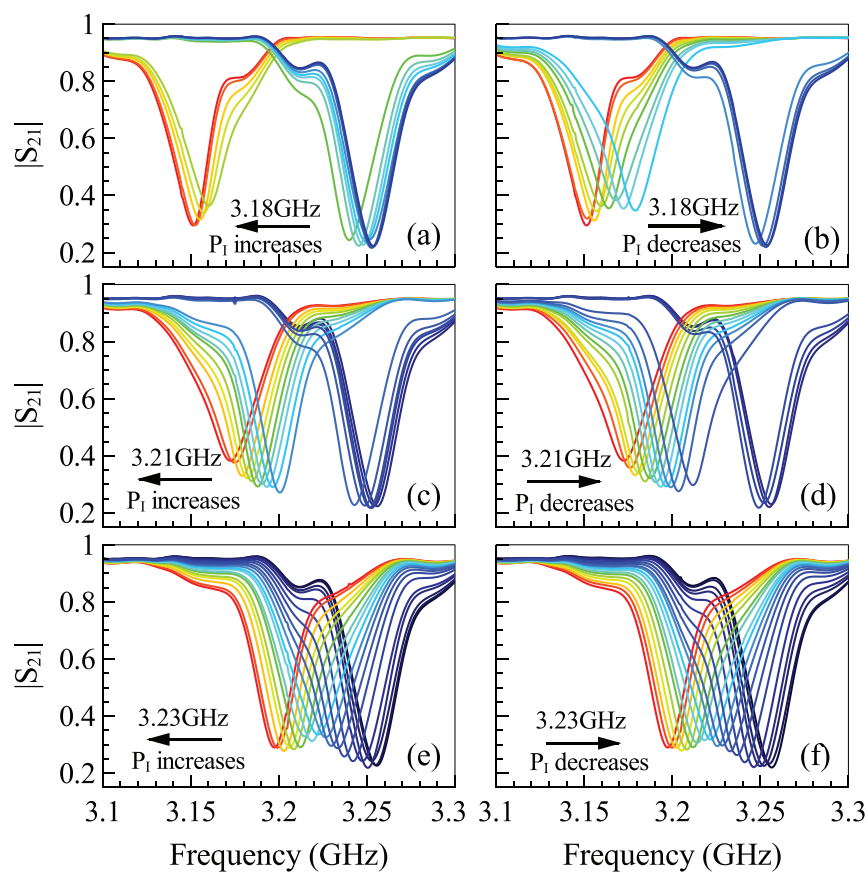

FIG. 4. (Color online) Experimental transmission coefficients $\left|S_{21}\right|$ for different pump frequencies and powers. The initial resonance is located around $3.256 \mathrm{GHz}$ and the pump power is swept in 1-dB steps. (a) and (b) Pump at $3.18 \mathrm{GHz}$, the power changes from 15.2 to $27.2 \mathrm{dBm}$; (c) and (d) pump at $3.21 \mathrm{GHz}$, the power changes from 12.2 to $27.2 \mathrm{dBm}$; (d) and (e) pump at $3.23 \mathrm{GHz}$, the power changes from 15.2 to $27.2 \mathrm{dBm}$. 

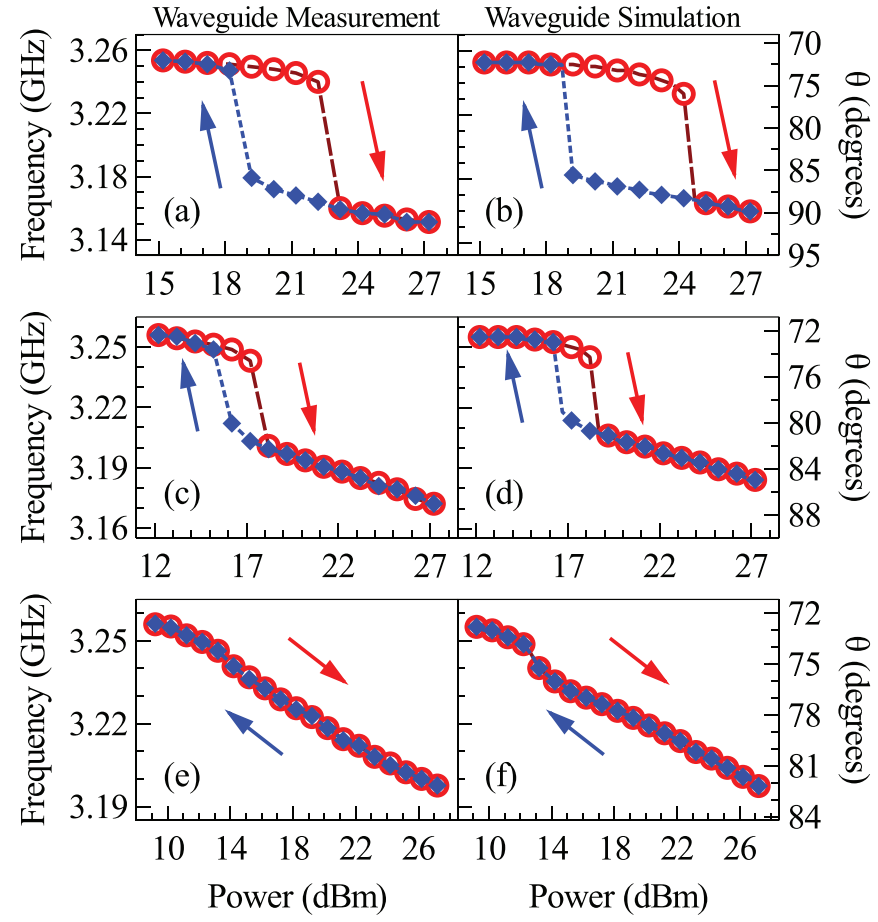

FIG. 5. (Color online) Comparison of experimentally [(a), (c), and (e)] and numerically [(b), (d), and (f)] calculated resonant frequency sweeps for identical geometry. The corresponding stable twist angles are shown on the right axes. (a) and (b) Pump at $3.18 \mathrm{GHz}$; (c) and (d) pump at $3.21 \mathrm{GHz}$; (e) and (f) pump at $3.23 \mathrm{GHz}$.

the pump frequency approaches to the initial resonance, the spectral "jump" becomes smaller [see Fig. 5(c)] and finally disappears [see Fig. 5(e)]. We also observed similar effects (not shown) when the pump frequency is at the red tail of the antisymmetric mode, in which case the two resonances approach each other due to the opposite direction of the EM torque.

\section{B. Comparison with simulation and discussion}

To further validate the observed effect, we numerically simulated the exact experimental geometry by taking into account the effect of the waveguide. The field distribution is calculated numerically using CST Microwave Studio, and the EM torque exerted on the top SRR is calculated with the Maxwell stress tensor, as shown in Fig. 6(a). Once the twist angle-dependent torque is known, the power-dependent twist angle can be found by solving the nonlinear equation (11). The estimated optimum initial twist angle is around $72.5^{\circ}$, and the maximum twist angles obtained for the three pump frequencies $(3.18,3.21$, and $3.23 \mathrm{GHz})$ are around $90^{\circ}, 85^{\circ}$, and $82^{\circ}$, respectively. Finally, we remap these angles back to the corresponding resonant frequencies according to the simulation spectra. We found excellent agreement between the measurement and simulation [see Figs. 5(b), 5(d), and 5(f)], thus confirming that our numerical model has reproduced the experimental configuration with acceptable accuracy.

Due to the formation of image currents within the waveguide walls, a single meta-atom within a waveguide has virtual neighbors. Just as in an array, there will be near-field

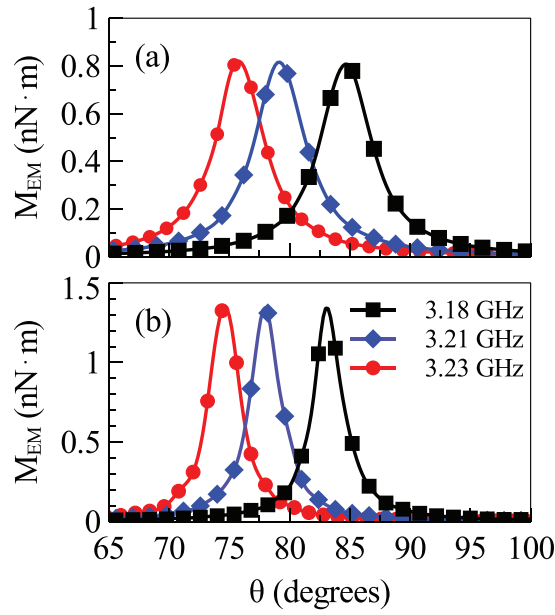

FIG. 6. (Color online) Mutual EM torque as a function of twist angle under different pump frequencies. (a) Single chiral meta-atom in waveguide and (b) periodic array of chiral meta-atoms, with periodicity $45 \mathrm{~mm}$ in the lateral direction and one layer in the propagating direction of EM wave. The torques shown correspond to a $1-\mathrm{W}$ power pumped into the waveguide or one unit cell.

interaction with these virtual neighbors, with some alteration due to the mirror reflections. This suggests that the waveguide system is strongly analogous to an array, at least for relatively dilute lattice spacing. To verify this, we numerically model the electromagnetic torque of the system for both the waveguide and array geometries. As shown in Fig. 6, the qualitative agreement between the two is very good.
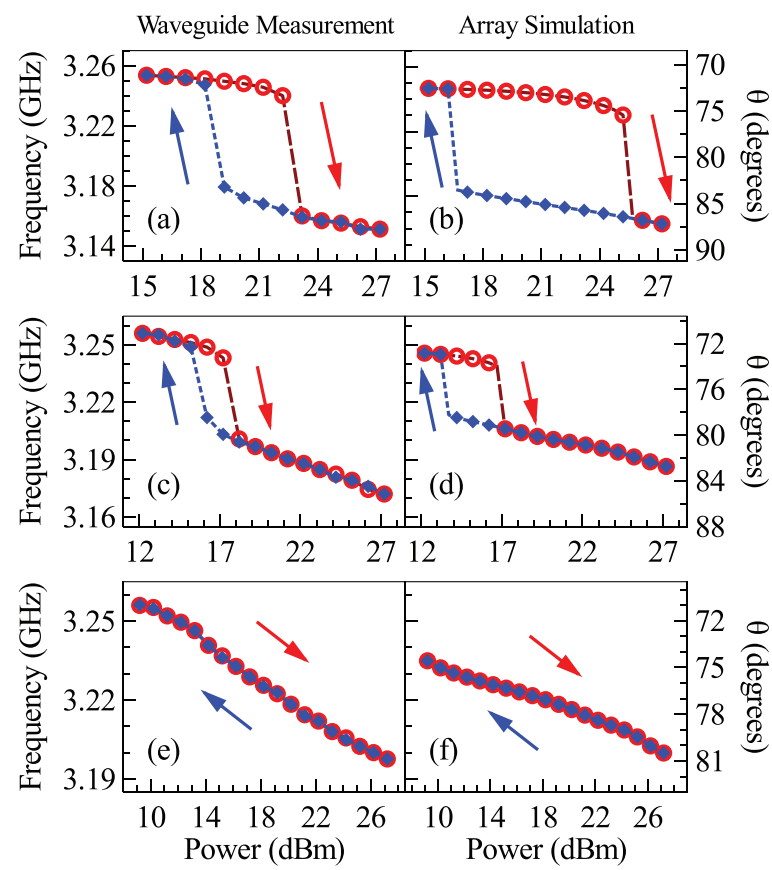

FIG. 7. (Color online) Comparisons of resonant frequencies demonstrated in a waveguide experiment and numerically calculated for an array. (a) and (b) Pump at $3.18 \mathrm{GHz}$; (c) and (d) pump at $3.21 \mathrm{GHz}$; (e) and (f) pump at $3.23 \mathrm{GHz}$. The stable twist angles for the array system are shown on the right axes. The power in the array is the power incident on each unit cell. 
Since the nonlinearity arises from individual rotatable metaatoms, the behavior in an array will not show qualitative difference from a single meta-atom as predicted in the analytical model, as long as the neighbor interaction is relatively weak. We use full numerical simulation to model an array with thickness of a single cell and periodicity of $45 \mathrm{~mm}$ in the transverse directions, arranged as in Fig. 1. The parameters of the metaatoms used in the simulation are the same as in the experiment. The array and the waveguide experiment show quite good qualitative agreement [see Figs. 7(b), 7(d), and 7(f) for the powerdependent resonance and Fig. 6 for the EM torque], thus justifying that the nonlinear behavior observed in the waveguide experiment is similar to that of the analogous array system.

\section{CONCLUSION}

We proposed and verified experimentally an interesting concept for achieving strong nonlinear coupling between the electromagnetic and elastic properties in metamaterials. The nonlinear "meta-atom" described in this work proved to possess a sensitive elastic feedback bringing nonlinearity to the interaction of EM modes of the resonators. The resulting nonlinearity and bistability of the response were successfully observed in experiments and it turns out that these results can be accurately predicted with theoretical modeling. We also note that this structure is chiral (except in the high symmetry cases of $0^{\circ}$ and $180^{\circ}$ angle between the rings), it should also exhibit nonlinear optical activity, an effect which is relatively weak in natural media, ${ }^{24}$ but can be quite strong in metamaterials. ${ }^{25}$

Although the experimental demonstration in this work was performed in the microwave frequency range, we expect that the general principle of operation is valid at any frequency where a resonant response can be excited in such or similar metamaterials elements, e.g., in the recently developed plasmonic chiral molecules based on DNA templates, ${ }^{26}$ and the way to analyze the same phenomena in $\mathrm{THz}$ or optical range is conceptually the same. We believe that this work provides a substantial contribution to the emerging area of optomechanical and magnetoelastic metamaterials, and offers an efficient and convenient design for practical applications.

\section{ACKNOWLEDGMENTS}

This work was supported by Australian Research Council. The authors are grateful to A. A. Sukhorukov for helpful discussions.
*Corresponding author: lmk124@physics.anu.edu.au

${ }^{1}$ L. Solymar and E. Shamonina, Waves in Metamaterials (Oxford University Press, 2009).

${ }^{2}$ R. Marqués, F. Martín, and M. Sorolla, Metamaterials with Negative Parameters (Wiley, 2008).

${ }^{3}$ D. R. Smith, W. J. Padilla, D. C. Vier, S. C. Nemat-Nasser, and S. Schultz, Phys. Rev. Lett. 84, 4184 (2000).

${ }^{4}$ J. B. Pendry, Phys. World 14, 47 (2001).

${ }^{5}$ V. M. Shalaev, Nat. Photon. 1, 41 (2007).

${ }^{6}$ J. Li and C. T. Chan, Phys. Rev. E 70, 055602 (2004).

${ }^{7}$ A. N. Norris, Proc. Royal Soc. A 464, 2411 (2008).

${ }^{8}$ Z. Nicolaou and A. Motter, Nat. Mater. 11, 608 (2012).

${ }^{9}$ M. Lapine et al., Appl. Phys. Lett. 95, 084105 (2009).

${ }^{10}$ A.-Q. Liu, W. M. Zhu, D. P. Tsai, and N. I. Zheludev, J. Opt. 14, 114009 (2012).

${ }^{11}$ M. Lapine, I. V. Shadrivov, D. A. Powell, and Y. S. Kivshar, Nat. Mater. 11, 30 (2012).

${ }^{12}$ M. Lapine, I. Shadrivov, and Y. Kivshar, Sci. Rep. 2, 412 (2012).

${ }^{13}$ F. Marquardt, and S. M. Girvin, Physics 2, 40 (2009).

${ }^{14}$ M. Lapine, I. V. Shadrivov, D. A. Powell, and Y. S. Kivshar, Sci. Rep. 1, 138 (2011).
${ }^{15}$ D. A. Powell, M. Lapine, M. V. Gorkunov, I. V. Shadrivov, and Y. S. Kivshar, Phys. Rev. B 82, 155128 (2010).

${ }^{16}$ D. A. Powell, K. Hannam, I. V. Shadrivov, and Y. S. Kivshar, Phys. Rev. B 83, 235420 (2011).

${ }^{17}$ H. Liu et al., Phys. Status Solidi B 246, 1397 (2009).

${ }^{18}$ P. N. Lebedev, Ann. der Physik 6, 433 (1901).

${ }^{19}$ M. Liu, D. A. Powell, and I. V. Shadrivov, Appl. Phys. Lett. 101, 031105 (2012).

${ }^{20}$ M. Liu, D. A. Powell, I. V. Shadrivov, and Y. S. Kivshar, Appl. Phys. Lett. 100, 111114 (2012).

${ }^{21}$ M. L. Povinelli et al., Opt. Lett. 30, 3042 (2005).

${ }^{22}$ J. D. Jackson, Classical electrodynamics (Wiley, New York, 1999).

${ }^{23}$ A. N. Gent, Engineering with Rubber: How to Design Rubber Components (Hanser Gardner Pubns, 2001).

${ }^{24}$ S. Kielich, Phys. Lett. A 25, 517 (1967).

${ }^{25}$ I. V. Shadrivov, V. A. Fedotov, D. A. Powell, Y. S. Kivshar, and N. I. Zheludev, New J. Phys. 13, 033025 (2011).

${ }^{26}$ S. J. Tan, M. J. Campolongo, D. Luo, and W. Cheng, Nat. Nanotechnology 6, 268 (2011). 